\title{
Mucoceles of the appendix mimicking ovarian cysts
}

\author{
Philipp Pecnik ${ }^{1}$, Regina Promberger ${ }^{2}$, Johannes Ott ${ }^{1}$ \\ 1. Department of Gynecologic Endocrinology and Reproductive Medicine, Medical University of Vienna, Vienna, Austria. 2. \\ Department of Surgery, Medical University of Vienna, Vienna, Austria \\ Correspondence: J ohannes Ott. Address: Department of Gynecologic Endocrinology and Reproductive Medicine, Medical \\ University of Vienna, Vienna, Austria. Telephone: 43-140-400-2816. Fax: 43-140-400-2817. \\ Email: johannes.ott@meduniwien.ac.at
}

Received: July 31, 2012

DOI : $10.5430 /$ jst.v2n5p54
Accepted: August 28, 2012

Published: October 1, 2012

\section{Abstract}

Background: This review focuses on appendiceal mucoceles that have been reported to incidentally mimic ovarian cysts and put the patient at risk of misdiagnosis and, thus, inadequate treatment.

Methodology: Review of the literature.

Principal findings: Benign mucoceles are the most common form of appendiceal mucoceles and represent about 63-84\%, whereas a malignancy is found in 11-20\%. Patient presentation is extremely variable for all forms, with, first and foremost, nonspecific symptoms, and patients may also be completely asymptomatic. Thus, the majority of appendiceal mucoceles are discovered incidentally. Women with ovarian cysts also frequently report abdominal pain or nonspecific gastrointestinal symptoms, with about $10 \%$ of patients being asymptomatic. Gynecologists perform transvaginal ultrasound as a standard procedure to evaluate pelvic tumors from the uterus, tubes, or ovaries. The ultrasound appearance of appendiceal mucoceles can vary widely from well-encapsulated purely cystic lesions with anechoic fluid, hypoechoic masses with fine internal echoes, or complex hyperechoic masses. Thus, they can mimic ovarian cysts. However, a specific ultrasonographic marker is the so-called "onion skin sign” and its "dumbbell structure”. Even when an appendiceal mucocele is detected during gynecologic surgery, the appropriate surgical treatment would be an open approach, ideally combined with an intraoperative frozen section examination. The gynecologist might choose not to continue with the operation, instead performing the excision of the appendiceal mucocele as a two-step procedure.

Conclusion/Significance: We advise gynecologists to consider the possibility of an appendiceal neoplasm, especially when a dumbbell structure in the lower right abdomen is found on ultrasound. If an appendiceal mucocele is incidentally diagnosed during surgery, a laparotomic approach is recommended if the operation is continued. This should be performed by a general surgeon.

\section{Key words}

Appendiceal mucocele, Appendiceal tumor, Ovarian cyst, Laparoscopy, Appendectomy, Sonography 


\section{Epidemiology}

Tumors of the appendix are infrequent, with an incidence of up to $2 \%$, and when they are found, it is almost always during a routine appendectomy ${ }^{[1,2]}$. Tumors of the appendix include leiomyomas, neuromas, lipomas, carcinoids, adenocarcinomas, and mucoceles among others ${ }^{[3]}$.

This review focuses on appendiceal mucoceles that have been reported to incidentally mimic ovarian cysts and put the patient at risk for misdiagnosis and, thus, inadequate treatment in the course of gynecologic surgery. Based on data from pathologic specimens, appendiceal mucoceles are rare conditions, with an incidence of approximately $0.07 \%$ to $0.3 \%{ }^{[4-6]}$. Concerning etiology, fecaliths may cause an acute luminal obstruction with secondary infection, thereby presenting as appendicitis, while hyperplasia or appendiceal tumors of the proximal lumen are long-term processes that may lead to mucocele formation rather than an acute inflammation. The etiology of the obstruction may not be apparent pre- or intraoperatively. Postoperative histology, however, usually resolves the obstructive etiology ${ }^{[5]}$.

\section{Methodology}

We searched Medline and EMBASE (search date: June 1st 2012; search terms: "mucocele" and "appendix" and/or "ovarian cyst") to identify case reports, case series, cohort studies, and reviews, reporting appendiceal mucoceles that mimic ovarian cysts and general information on the epidemiology, histopathology, diagnosis and therapy of appendiceal mucoceles. Studies were included if they were published as complete reports in English. Bibliographies of studies were searched for relevant citations.

\section{Histopathologic aspects}

There are four different subgroups of mucoceles, defined on the basis of their histologic characteristics. An overview is provided in Table $1^{[5,7-9]}$. The first type, a simple retention cyst, is characterized by normal histology and mild luminal dilatation that rarely exceeds more than $2 \mathrm{~cm}$ in diameter ${ }^{[10]}$.

Table 1. Histologic subgroups of appendiceal mucoceles

\begin{tabular}{|c|c|}
\hline Appendiceal tumor & Histological signs \\
\hline $\begin{array}{l}\text { Retention cyst / simple mucocele / simple } \\
\text { ectasia }\end{array}$ & $\begin{array}{l}\text { The mucosal lining in this group is either normal or may contain inflammatory cells. } \\
\text { Proximal obstruction is usually caused by a fecalith, leading to distention of the lumen } \\
\text { with mucous. Most presentations are consistent with acute appendicitis. }\end{array}$ \\
\hline Mucosal hyperplasia & $\begin{array}{l}\text { Benign hyperplasia of the cells lining the appendix denotes obstruction of the proximal } \\
\text { appendix. The course is usually insidious due to the chronic nature of the obstruction. }\end{array}$ \\
\hline Cystadenoma & $\begin{array}{l}\text { Adenomatous epithelial lining of the appendix with either focal or diffuse conversion } \\
\text { of the appendiceal mucosa into neoplastic epithelium The mucosal lining of } \\
\text { cystadenomas is identical to adenomatous polyps and papillary adenomas of the colon. } \\
\text { Cystadenomas usually present as a chronic non-infectious process. }\end{array}$ \\
\hline Cystadenocarcinomas & $\begin{array}{l}\text { An invasive adenocarcinoma causes obstruction of the lumen. Stromal invasion by } \\
\text { neoplastic cells distinguishes cystadenomas and cystadenocarcinomas. }\end{array}$ \\
\hline
\end{tabular}

A diameter over $2 \mathrm{~cm}$ increases the likelihood that the cyst is one of the other three types of mucoceles ${ }^{[10]}$. The second subgroup, accounting for $5-25 \%$ of mucoceles, is also characterized by mild dilatation of the appendiceal lumen, but regions of hyperplastic epithelium are found in histologic specimens ${ }^{[11]}$.

Benign mucoceles, the third and most common form, represent about $63-84 \%$ of all appendiceal mucoceles. The lumen is distended by up to $6 \mathrm{~cm}$ in diameter, and the epithelium is similar to villous adenomas and adenomatous polyps with 
low-grade dysplasia, as found in the colon ${ }^{[10,11]}$. In mucinous cystadenomas, spontaneous rupture of the appendix may occur in about $20 \%$ of cases, leading to spillage on the peritoneum and surrounding visceral surfaces. After spontaneous or intraoperative penetration of a benign cystadenoma, a few cases of pseudomyxoma peritonei have been reported, which were limited to the peri-appendicular area ${ }^{[11,12]}$. Patients with a non-neoplastic or a benign neoplastic mucocele show a five-year survival prognosis of $91-100 \%{ }^{[10]}$.

The fourth subgroup is the malignant mucinous cystadenocarcinoma, representing 11-20\% of patients with mucoceles. Compared to cystadenomas, cystadenocarcinomas show stromal invasion, and, under severe conditions, even malignant cell implants on the peritoneum, the colon, and/or the ovaries ${ }^{[9,13]}$. The appendiceal lumen may be distended massively. Mucinous cystadenocarcinomas might show spontaneous rupture in $6 \%$ of cases, leading to pseudomyxoma peritonei. In this condition, the peritoneal cavity becomes stained with semi-solid mucin, in which malignant cells of the cystadenocarcinoma can be found ${ }^{[11]}$. The intraperitoneal spread of the carcinoma is identical to that of intraperitoneal ovarian mucinous cystadenocarcinomas ${ }^{[14]}$. Lymph node or liver metastasis is rarely found ${ }^{[15]}$. Due to complications, pseudomyxoma peritonei significantly decreases the five-year survival rate of patients with a malignant neoplasia to around $25 \%{ }^{[11,14]}$. In $11-20 \%$, an appendiceal mucinous cystadenocarcinoma is associated with other tumors, particularly colon carcinoma and ovarian carcinoma ${ }^{[16]}$.

\section{Clinical features}

Appendiceal mucoceles are more common in women, with a female-to-male ratio of about 4:1. Age over 50 years has been reported as a second risk factor ${ }^{[13]}$. For all of the subgroups, patient presentation is extremely variable, and patients may also be completely asymptomatic. Thus, the majority of appendiceal mucoceles are discovered incidentally ${ }^{[5,7,13]}$.

Specifically, 25-50\% of patients with a mucocele are asymptomatic, whereas the remaining $50-75 \%$ reveals nonspecific symptoms, including mild to moderate abdominal pain, which is likely due to distention of the mucus-filled appendix, weight loss, nausea, and emesis, in descending order ${ }^{[17,18]}$. Rarer conditions include right ureteric and/or bowel obstruction, intussusception, torsion, or gastrointestinal bleeding ${ }^{[10,19]}$. A palpable mass can be found in $50 \%$ of cases. Ascites and an increase in the abdominal circumference may be diagnosed due to the progression of a malignant entity ${ }^{[15]}$.

The literature on clinical symptoms of ovarian cysts is sparse. In recent reports, women with ovarian cysts or masses frequently reported abdominal pain or nonspecific gastrointestinal symptoms. About $10 \%$ of patients were asymptomatic ${ }^{[20,21]}$. Thus, the symptom load seems quite comparable to that of appendiceal mucoceles. It is reasonable that if signs and symptoms are shared by both conditions, the differential diagnosis is more difficult. However, it has been suggested that an appendiceal mucocele should be considered in women with a right-sided ovarian cyst and clinical features not indicative of gynecological pathology, including nausea, vomiting, and altered bowel habits ${ }^{[22]}$.

\section{Diagnosis}

When focusing on appendiceal mucoceles mimicking ovarian cysts, it should be noted that diagnosis is likely based on gynecologic ultrasound. Gynecologists perform transvaginal ultrasound as a standard procedure to evaluate pelvic tumors from the uterus, tubes, or ovaries. Masses that do not originate from the female reproductive organs can be misjudged and be interpreted as ovarian cysts ${ }^{[23]}$. It has been suggested that abdominal ultrasound does not increase the rate of correct diagnosis ${ }^{[24]}$. Ultrasound findings can vary widely. Depending on the contents, well-encapsulated purely cystic lesions with anechoic fluid, hypoechoic masses with fine internal echoes, or complex hyperechoic masses can be seen ${ }^{[17,25,26]}$. According to the ultrasound-based guidelines that define a tumor as benign (B-rules) or malignant (M-rules) in ovarian masses ${ }^{[27]}$, the vast majority of appendiceal mucoceles mimicking ovarian cysts would probably be classified as "benign" as they, for the most part, fulfill the following criteria: (i) unilocular cyst; (ii) presence of solid components where the 
largest solid component is $<7 \mathrm{~mm}$ at the largest diameter; (iii) acoustic shadows; (iv) smooth multilocular tumor less than $100 \mathrm{~mm}$ at the largest diameter; and (v) no detectable blood flow on Doppler examination ${ }^{[27]}$.

However, a specific ultrasonographic marker of an appendiceal mucocele is the so-called "onion skin sign" and its "dumbbell structure" ${ }^{[17,23]}$. The onion-skin sign can also be found in other mucinous cysts, such as the ovaries, which can thus make diagnosis more difficult ${ }^{[28]}$. This sign has been described as concentric echogenic layers that include numerous septa and fine gravity-dependent echoes ${ }^{[28]}$.

Additional radiologic diagnostic tools might be helpful for the diagnosis of an appendiceal mucocele. Computer tomography (CT) of the lower abdomen usually shows a dumbbell-structured, cystic and well-encapsulated mass, including mural calcification in less than $50 \%{ }^{[10,17,23]}$. An intravenous contrast enema helps depict local blood vessels. Using a barium enema, the contrast medium usually fills the appendix only partially ${ }^{[17]}$. Cystic masses described with magnetic resonance tomography (MRT) show low signal intensity on T1-weighted images and high signal intensity on T2-weighted images ${ }^{[29]}$. Both CT and MRT are useful additional examinations that are notable for their excellent evaluation of tissue characteristics and exact anatomic location of masses. MRT, in particular, can be used to depict local inflammation and differentiate cysts from the female reproductive organs to exclude the possibility of ovarian cysts. MRT also has the advantage of emitting no radiation. However, if a simple benign ovarian cyst is suspected, neither CT nor MRT are considered useful ${ }^{[30]}$. Thus, CT and MRT are unlikely to be indicated in the evaluation of appendiceal mucoceles.

The same is true for colonoscopy. If a colonoscopy is performed, the "volcano sign" is indicative of an appendiceal mucocele, with the orifice of the appendix lying in the center of a mound, covered by normal mucosa or a yellowish, lipomatous submucosal mass ${ }^{[17]}$. It then is advisable not to move the colonoscope all the way into the cecum to prevent rupture and peritoneal seeding.

In premenopausal women with simple ovarian cysts whose symptoms meet the B-rules guidelines, determination of tumor markers is considered unnecessary. CA 125 levels might be obtained only in postmenopausal women ${ }^{[30]}$. However, tumor markers, including CEA, CA 19-9, and CA 125 have not proven to be parameters on which to build the diagnosis of appendiceal tumors, as cases of false-negative tumor marker levels in patients with cystadenomas have been reported $^{[17,29,31]}$.

Taking all these considerations together, the definitive diagnosis of a neoplasm can be made only postoperatively by histopathology; intraoperatively, frozen section examination can provide diagnosis in many cases, but are distinguished by lower quality than formalin fixed slides ${ }^{[32,33]}$.

\section{Treatment}

If an appendiceal mucocele is misdiagnosed as an ovarian cyst, a surgical intervention is likely because of persistence or even growth of the detected intraabdominal mass. In this setting, the woman will undergo gynecologic laparoscopy ${ }^{\text {[34] }}$. This procedure should reveal the structure's origin. But what is the correct procedure following the intraoperative diagnosis of an appendiceal mucocele in the course of gynecologic surgery?

Surgical excision can be made by either laparoscopy or laparotomy. Laparoscopic surgery provides the advantages of good exposure of the entire abdominal cavity, rapid recovery, and a better cosmetic outcome ${ }^{[17]}$. On the other hand, careful handling of the mucinous appendix is indicated to prevent spillage into the peritoneal cavity, which gives the laparotomic surgery procedure an advantage. If malignancy is suspected, the transition from a laparoscopic to a laparotomic 
exploration has been recommended in order to minimize the risk of rupture and peritoneal extension ${ }^{[10]}$. Furthermore, Bucher et al. proved a slightly increased rate of inadequate resection when laparoscopic appendectomy was performed, with unclear surgical margins (R1-resection) and a higher rate of peritoneal seeding ${ }^{[1,35]}$.

Thus, the appropriate surgical treatment is an open approach combined with an intraoperative frozen section examination ${ }^{[33]}$. A simple retention cyst, an appendix with epithelial hyperplasia, and a cystadenoma with an intact base are treated by simple appendectomy ${ }^{[34,36]}$. Cystadenomas with a larger base of implantation are an indication for cecum resection. Right hemicolectomy used to be the standard treatment for cystadenocarcinomas, but studies have proven no survival advantages with this procedure ${ }^{[37,38]}$. A right hemicolectomy is indicated only if one of the following three criteria apply: (i) necessity to clear the primary tumor or achieve complete cytoreduction; (ii) lymph node involvement; or (iii) histopathological examination indicating a non-mucinous type of cyst ${ }^{[34,36]}$.

Simple retention cysts and benign neoplasms show a five-year survival rate of 91-100\%, whereas the five-year survival rate for malignant neoplasms is only $25 \%$, due to the complication of pseudomyxoma peritonei ${ }^{[13]}$. Moreover, during laparotomy, an accurate examination of the abdominal cavity is recommended since there is an association between mucinous appendix and colon carcinoma in $11-20 \%{ }^{[10,11]}$. As an example, treatment of an associated ovarian cystadenocarcinoma includes hemicolectomy, bilateral oophorectomy, and omentectomy ${ }^{[33]}$.

The incidental finding of an appendiceal mucocele also presents a forensic problem, since it is unlikely that the patient has been informed about gastrointestinal surgery in most cases. The gynecologist might choose not to continue with the operation but rather, to perform the excision of the appendiceal mucocele as a two-step procedure. If surgery is continued, it is recommended that this should be performed by a general surgeon ${ }^{[23]}$.

\section{Conclusion}

Appendiceal mucoceles are typically found in middle-aged patients, with a higher incidence in women. Patients often present with nonspecific symptoms and can also be asymptomatic. In some cases, the mucocele is diagnosed during routine gynecologic sonography and can be misdiagnosed as ovarian cysts. We advise gynecologists to consider the possibility of an appendiceal neoplasm, especially when a dumbbell structure in the lower right abdomen is found on ultrasound. If an appendiceal mucocele is incidentally diagnosed during surgery, a laparotomic approach is recommended if the operation is continued. A general surgeon should perform such surgery.

\section{References}

[1] P. Bucher, Z. Mathe, a Demirag, and P. Morel. Appendix tumors in the era of laparoscopic appendectomy. Surgical endoscopy. 2004 Jul.; 18(7): 1063-6. PMid:15156378 http://dx.doi.org/10.1007/s00464-003-9255-X

[2] D. Collins. 71,000 human appendix specimens. a final report, summarizing forty years’ study. Am J Proctol. 1963; 14(365).

[3] A. Uihlein and J. R. McDonald. Primary carcinoma of the appendix resembling carcinoma of the colon. Surg Gynecol Obstet. 1943; 76: 711.

[4] A. Warren and A. Warren. A study of 6797 surgically removed appendices. Ann Surg. 1926; 83: 222-235. PMid:17865412 http://dx.doi.org/10.1097/00000658-192602000-00010

[5] R. Woodruff and J. McDonald. Benign and malignant cystic tumors of the appendix. Surg Gynecol Obstet. 1940 ; 71: $750-755$.

[6] O. Castle. Cystic dilation of the vermiform appendix. Ann Surg. 1915; 61: 582. PMid:17863360 http://dx.doi.org/10.1097/00000658-191505000-00007

[7] M. Steinberg and I. Cohn. Primary adenocarcinoma of the appendix. Surgery. 1967; 61: 644-660. PMid:6020971

[8] A. Qizilbash. Primary adenocarcinoma of the appendix: A clinicopathological study of 11 cases. Arch Pathol. $1975 ; 99: 556-562$. PMid:172053 
[9] E. Higa, J. Rosai, C. A. Pizzimbono, and L. Wise. Mucosal hyperplasia, mucinous cystadenoma, and mucinous cystadenocarcinoma of the appendix. A re-evaluation of appendiceal 'mucocele'. Cancer. 1973 Dec.; 32(6): $1525-41$. http://dx.doi.org/10.1002/1097-0142(197312)32:6<1525::AID-CNCR2820320632>3.0.CO;2-C

[10] T. Persaud, N. Swan, and W. Torreggiani. Giant Mucinous Cystadenoma of the Appendix. Radiographics. 2007 ; $553-557$. PMid:17374868 http://dx.doi.org/10.1148/rg.272065134

[11] B. Rampone, F. Roviello, D. Marrelli, and E. Pinto. Giant appendiceal mucocele: report of a case and brief review. World journal of gastroenterology : WJG. 2005 Aug.; 11(30): 4761-3. PMid:16094726

[12] A. Koktener, K. Akin, D. Kosehan, and C. Dener. Primary appendicealtumors: clinical imaging and pathological findings. Report of four cases. JBR-BTR : organe de la Société royale belge de radiologie (SRBR) = orgaan van de Koninklijke Belgische Vereniging voor Radiologie (KBVR).2011 Mar-Apr; 94(2): 63-5.

[13] A. J. Aho, R. Heinonen, and P. Laurén. Benign and malignant mucocele of the appendix. Histological types and prognosis. Acta chirurgica Scandinavica. 1973 Jan.; 139(4): 392-400. PMid:4718184

[14] S. Landen et al. Appendiceal mucoceles and pseudomyxoma peritonei. Surgery, gynecology \& obstetrics. 1992 Nov.; 175(5): 401-4. PMid:1440166

[15] P. H. Sugarbaker. New standard of care for appendiceal epithelial neoplasms and pseudomyxoma peritonei syndrome? The lancet oncology. 2006 Jan.; 7(1): 69-76. http://dx.doi.org/10.1016/S1470-2045(05)70539-8

[16] M. Kahn and I. H. Friedman. Mucocele of the appendix: diagnosis and surgical management. Diseases of the colon and rectum. 1979; 22(4): 267-9. PMid:19213371 http://dx.doi.org/10.1007/BF02586892

[17] C. Bartlett, M. Manoharan, and A. Jackson. Mucocele of the appendix - a diagnostic dilemma: a case report. Journal of medical case reports. 2007 Jan.; 1: 183. PMid:18093329 http://dx.doi.org/10.1186/1752-1947-1-183

[18] C. Chiu, P. Wei, M. Huang, W. Wang, T. Chen, and W. Lee. Laparoscopic resection of appendiceal mucinous cystadenoma. J Laparoendosc Adv Surg Tech A. 2005; 15: 325-8. PMid:22743261 http://dx.doi.org/10.1089/lap.2005.15.325

[19] L. Stocchi, B. G. Wolff, D. R. Larson, and J. R. Harrington. Surgical treatment of appendiceal mucocele. Archives of surgery (Chicago, Ill. : 1960). 2003 Jun.; 138(6): 585-9; discussion 589-90.

[20] A. Guzel, U. Kuyumcuoglu, and M. Erdemoglu. Adnexal masses in postmenopausal and reproductive age women. J Exp Ther Oncol. 2011; 9(2): 167-9. PMid:21699024

[21] T. Wasim, A. Majrroh, and S. Siddiq. Comparison of clinical presentation of benign and malignant ovarian tumours. J Pak Med Assoc. 2009 Jan; 59(1): 18-21.

[22] K. Prasad, Shetty, M. Ramesh, Saraswathi, and Ramesh. Mucocele of Appendix Secondary to Cystadenoma a Diagnostic Challenge. OJHAS. 2010; 9 (2): 14.

[23] K. Nouri, M. Demmel, J. Ott, R. Promberger, J. C. Huber, and K. Mayerhofer. Villous mucinous cystadenoma of the appendix in a postmenopausal woman. JSLS : Journal of the Society of Laparoendoscopic Surgeons / Society of Laparoendoscopic Surgeons. 2010; 14(2): 296-8.

[24] C. C. Huang, L. K. Lo, and L. W. Koh. Pelviscopic removal of an appendiceal mucocele. The Journal of the American Association of Gynecologic Laparoscopists. 1994 Feb.; 1(2): 174-6. http://dx.doi.org/10.1016/S1074-3804(05)80786-8

[25] P. Skaane, T. E. Ruud, and J. Haffner, “Ultrasonographic features of mucocele of the appendix”. Journal of clinical ultrasound : JCU. 1998 Oct.; 16(8): 584-7. PMid:3152405

[26] B. H. R. Vriens and J. M. Klaase. Giant mucinous cystadenoma of the appendix. American journal of surgery. 2007 Sep.; 194(3): 392-3. PMid:17693289 http://dx.doi.org/10.1016/j.amjsurg.2006.11.041

[27] D. Timmerman et al. Simple ultrasound-based rules for the diagnosis of ovarian cancer. Ultrasound in obstetrics \& gynecology : the official journal of the International Society of Ultrasound in Obstetrics and Gynecology. 2008 Jun.; 31(6): 681-90.

[28] S. Perlman, Y. Hazan, Z. Hagay, Z. Appelman, and B. Caspi. “Onion skin” sign in an ovarian mucinous cyst. Journal of clinical ultrasound: JCU. Jan. 2012.

[29] I. Okuda, M. Matsuda, H. Noguchi, and T. Kokubo. Massive mucinous cystadenoma of the appendix with intussusception in an adult: usefulness of reconstructed computed tomography images. Radiation medicine. 2008 Feb.; 26(2): 88-91. PMid:18301985 http://dx.doi.org/10.1007/s11604-007-0201-z

[30] E. R. Myers et al. Management of adnexal mass. Evidence report/technology assessment. 2006 Feb.; 130: 1-145.

[31] C. Scaffa, O. Di Bella, E. Tartaglia, M. Rotondi, F. Lup, and E. M. Messalli. Surgical approach to appendiceal mucocele mimicking an adnexal complex mass: case report. European journal of gynaecological oncology. 2007 Jan.; 28(6): 503-5. PMid:18179147

[32] H. Iswariah, M. Metcalfe, D. Lituri, and G. Maddern. Mucinous cystadenoma of the appendix. ANZ journal of Surgery. 2004; January: 918-919. PMid:15456452 http://dx.doi.org/10.1111/j.1445-1433.2004.03180.x 
[33] A. Cois, A. Pisanu, L. Pilloni, and A. Uccheddu. Intussusception of the appendix by mucinous cystadenoma. Report of a case with an unusual clinical presentation. Chirurgia italiana. 2006; 58(1): 101-4. PMid:16729616

[34] D. Caracappa et al. Appendiceal mucocele. A case report and literature review. Annali italiani di chirurgia. 2011; 82(3): 239-45. PMid:21780569

[35] V. Paolucci, B. Schaeff, M. Schneider, and C. Gutt. Tumor seeding following laparoscopy: international survey. World journal of surgery. 1999 Oct.; 23(10): 989-95; discussion 996-7. PMid:10512937 http://dx.doi.org/10.1007/s002689900613

[36] A. Dixit, J. H. P. Robertson, S. S. Mudan, and C. Akle. Appendiceal mucocoeles and pseudomyxoma peritonei. World journal of gastroenterology: WJG. 2007 Apr.; 13(16): 2381-4. PMid:17511043

[37] S. González-Moreno and P. H. Sugarbaker. Right hemicolectomy does not confer a survival advantage in patients with mucinous carcinoma of the appendix and peritoneal seeding. The British journal of surgery. 2004 Mar.; 91(3): 304-11. PMid:14991630 http://dx.doi.org/10.1002/bjs.4393

[38] J. M. Foster et al. Right hemicolectomy is not routinely indicated in pseudomyxoma peritonei. The American surgeon. 2012 Feb.; 78(2): 171-7. PMid:22369825 